\title{
New Method for Solving Linear Fractional Differential Equations
}

\author{
S. Z. Rida and A. A. M. Arafa \\ Department of Mathematics, Faculty of Science, South Valley University, Qena 83523, Egypt \\ Correspondence should be addressed to A. A. M. Arafa, anaszi2@yahoo.com
}

Received 4 May 2011; Revised 21 July 2011; Accepted 25 July 2011

Academic Editor: Shaher M. Momani

Copyright (C) 2011 S. Z. Rida and A. A. M. Arafa. This is an open access article distributed under the Creative Commons Attribution License, which permits unrestricted use, distribution, and reproduction in any medium, provided the original work is properly cited.

We develop a new application of the Mittag-Leffler Function method that will extend the application of the method to linear differential equations with fractional order. A new solution is constructed in power series. The fractional derivatives are described in the Caputo sense. To illustrate the reliability of the method, some examples are provided. The results reveal that the technique introduced here is very effective and convenient for solving linear differential equations of fractional order.

\section{Introduction}

Fractional differential equations have excited, in recent years, a considerable interest both in mathematics and in applications. They were used in modeling of many physical and chemical processes and engineering (see, e.g., [1-6]). In its turn, mathematical aspects of fractional differential equations and methods of their solutions were discussed by many authors: the iteration method in [7], the series method in [8], the Fourier transform technique in $[9,10]$, special methods for fractional differential equations of rational order or for equations of special type in [11-16], the Laplace transform technique in [3-6, 16, 17], and the operational calculus method in [18-23]. Recently, several mathematical methods including the Adomian decomposition method [18-25], variational iteration method [23-26] and homotopy perturbation method $[27,28]$ have been developed to obtain the exact and approximate analytic solutions. Some of these methods use transformation in order to reduce equations into simpler equations or systems of equations, and some other methods give the solution in a series form which converges to the exact solution.

The reason of using fractional order differential (FOD) equations is that FOD equations are naturally related to systems with memory which exists in most biological systems. Also they are closely related to fractals which are abundant in biological systems. The results 
derived from the fractional system are of a more general nature. Respectively, solutions to the fractional diffusion equation spread at a faster rate than the classical diffusion equation and may exhibit asymmetry. However, the fundamental solutions of these equations still exhibit useful scaling properties that make them attractive for applications.

The concept of fractional or noninteger order derivation and integration can be traced back to the genesis of integer order calculus itself [29]. Almost all of the mathematical theory applicable to the study of noninteger order calculus was developed through the end of the 19th century. However, it is in the past hundred years that the most intriguing leaps in engineering and scientific application have been found. The calculation techniques in some cases meet the requirement of physical reality. The use of fractional differentiation for the mathematical modeling of real-world physical problems has been widespread in recent years, for example, the modeling of earthquake, the fluid dynamic traffic model with fractional derivatives, and measurement of viscoelastic material properties. Applications of fractional derivatives in other fields and related mathematical tools and techniques could be found in [30-41]. In fact, real-world processes generally or most likely are fractional order systems.

The derivatives are understood in the Caputo sense. The general response expression contains a parameter describing the order of the fractional derivative that can be varied to obtain various responses.

\section{Fractional Calculus}

There are several approaches to the generalization of the notion of differentiation to fractional orders, for example, the Riemann-Liouville, Grünwald-Letnikov, Caputo, and generalized functions approach [42]. The Riemann-Liouville fractional derivative is mostly used by mathematicians but this approach is not suitable for real-world physical problems since it requires the definition of fractional order initial conditions, which have no physically meaningful explanation yet. Caputo introduced an alternative definition, which has the advantage of defining integer order initial conditions for fractional order differential equations [42]. Unlike the Riemann-Liouville approach, which derives its definition from repeated integration, the Grünwald-Letnikov formulation approaches the problem from the derivative side. This approach is mostly used in numerical algorithms.

Here, we mention the basic definitions of the Caputo fractional-order integration and differentiation, which are used in the upcoming paper and play the most important role in the theory of differential and integral equation of fractional order.

The main advantages of Caputo approach are the initial conditions for fractional differential equations with the Caputo derivatives taking on the same form as for integer order differential equations.

Definition 2.1. The fractional derivative of $f(x)$ in the Caputo sense is defined as

$$
\begin{aligned}
D^{\alpha} f(x) & =I^{m-\alpha} D^{m} f(x) \\
& =\frac{1}{\Gamma(m-\alpha)} \int_{0}^{x}(x-t)^{m-\alpha+1} f^{(m)}(t) d t
\end{aligned}
$$

for $m-1<\alpha \leq m, m \in N, x>0$. 
For the Caputo derivative we have $D^{\alpha} \mathrm{C}=0, \mathrm{C}$ is constant,

$$
D^{\alpha} t^{n}=\left\{\begin{array}{ll}
0, & (n \leq \alpha-1), \\
\frac{\Gamma(n+1)}{\Gamma(n-\alpha+1)} t^{n-\alpha}, & (n>\alpha-1) .
\end{array}\right\} .
$$

Definition 2.2. For $m$ to be the smallest integer that exceeds $\alpha$, the Caputo fractional derivative of order $\alpha>0$ is defined as

$$
\begin{aligned}
D^{\alpha} u(x, t) & =\frac{\partial^{\alpha} u(x, t)}{\partial t^{\alpha}} \\
& =\left\{\begin{array}{ll}
\frac{1}{\Gamma(m-\alpha)} \int_{0}^{t}(t-\tau)^{m-\alpha+1} \frac{\partial^{m} u(x, \tau)}{\partial \tau^{m}} d \tau, & \text { for } m-1<\alpha<m \\
\frac{\partial^{m} u(x, t)}{\partial t^{m}}, & \text { for } \alpha=m \in N
\end{array}\right\} .
\end{aligned}
$$

\section{Analysis of the Method}

The Mittag-Leffler (1902-1905) functions $E_{\alpha}$ and $E_{\alpha, \beta}$ [42], defined by the power series

$$
E_{\alpha}(z)=\sum_{k=0}^{\infty} \frac{z^{k}}{\Gamma(\alpha k+1)}, \quad E_{\alpha, \beta}(z)=\sum_{k=0}^{\infty} \frac{z^{k}}{\Gamma(\alpha k+\beta)}, \quad \alpha>0, \quad \beta>0,
$$

have already proved their efficiency as solutions of fractional order differential and integral equations and thus have become important elements of the fractional calculus theory and applications.

In this paper, we will explain how to solve some of differential equations with fractional level through the imposition of the generalized Mittag-Leffler function $E_{\alpha}(z)$. The generalized Mittag-Leffler method suggests that the linear term $y(x)$ is decomposed by an infinite series of components:

$$
y=E_{\alpha}\left(a x^{\alpha}\right)=\sum_{n=0}^{\infty} a^{n} \frac{x^{n \alpha}}{\Gamma(n \alpha+1)} .
$$

We will use the following definitions of fractional calculus:

$$
\begin{aligned}
D^{\alpha} y & =\sum_{n=1}^{\infty} a^{n} \frac{x^{(n-1) \alpha}}{\Gamma((n-1) \alpha+1)} \\
D^{2 \alpha} y & =\sum_{n=2}^{\infty} a^{n} \frac{x^{(n-2) \alpha}}{\Gamma((n-2) \alpha+1)}
\end{aligned}
$$

This is based on the Caputo fractional is derivatives. The convergence of the Mittag Leffler function discussed in [42]. 


\section{Applications and Results}

In this section, we consider a few examples that demonstrate the performance and efficiency of the generalized Mittag-Leffler function method for solving linear differential equations with fractional derivatives.

Example 4.1. Consider the following fractional differential equation [43]:

$$
\frac{d^{\alpha} y}{d x^{\alpha}}=A y
$$

By using (3.3) into (4.1) we find

$$
\sum_{n=1}^{\infty} a^{n} \frac{x^{(n-1) \alpha}}{\Gamma((n-1) \alpha+1)}-A \sum_{n=0}^{\infty} a^{n} \frac{x^{n \alpha}}{\Gamma(n \alpha+1)}=0
$$

Combining the alike terms and replacing $(n)$ by $(n+1)$ in the first sum, we assume the form

$$
\begin{gathered}
\sum_{n=0}^{\infty} a^{n+1} \frac{x^{n \alpha}}{\Gamma(n \alpha+1)}-A \sum_{n=0}^{\infty} a^{n} \frac{x^{n \alpha}}{\Gamma(n \alpha+1)}=0, \\
\sum_{n=0}^{\infty}\left(a^{n+1}-A a^{n}\right) \frac{x^{n \alpha}}{\Gamma(n \alpha+1)}=0 .
\end{gathered}
$$

With the coefficient of $x^{n \alpha}$ equal to zero and identifying the coefficients, we obtain recursive

$$
\begin{aligned}
& a^{n+1}-A a^{n}=0 \Longrightarrow a^{n+1}=A a^{n}, \\
& \text { at } n=0, \quad a^{1}=A a^{0}=A, \\
& \text { at } n=1, \quad a^{2}=A a^{1} \Longrightarrow a^{2}=A^{2}, \\
& \text { at } n=2, \quad a^{3}=A a^{2} \Longrightarrow a^{3}=A^{3} .
\end{aligned}
$$

Substituting into (3.2)

$$
\begin{aligned}
& y(x)=a^{0}+a^{1} \frac{x^{\alpha}}{\Gamma(\alpha+1)}+a^{2} \frac{x^{2 \alpha}}{\Gamma(2 \alpha+1)}+a^{3} \frac{x^{3 \alpha}}{\Gamma(3 \alpha+1)}+\cdots, \\
& y(x)=1+A \frac{x^{\alpha}}{\Gamma(\alpha+1)}+A^{2} \frac{x^{2 \alpha}}{\Gamma(2 \alpha+1)}+A^{3} \frac{x^{3 \alpha}}{\Gamma(3 \alpha+1)}+\cdots
\end{aligned}
$$

The general solution is

$$
y(x)=\sum_{n=0}^{\infty} \frac{A^{n} x^{n \alpha}}{\Gamma(n \alpha+1)} .
$$


We can write the general solution in the Mittag-Leffler function form as

$$
y(x)=E_{\alpha}\left(A^{n} x^{\alpha}\right)
$$

As $\alpha=1$, we have the exact solution:

$$
y(x)=\sum_{n=0}^{\infty} \frac{(A x)^{n}}{\Gamma(n+1)}=e^{A x}
$$

which is the exact solution of the standard form.

Example 4.2. Consider the fractional differential equation [44]

$$
\frac{d^{2 \alpha} y}{d x^{2 \alpha}}-y=0
$$

By using (3.2) and (3.4) into (4.9) we find

$$
\sum_{n=2}^{\infty} a^{n} \frac{x^{(n-2) \alpha}}{\Gamma((n-2) \alpha+1)}-\sum_{n=0}^{\infty} a^{n} \frac{x^{n \alpha}}{\Gamma(n \alpha+1)}=0
$$

Combining the alike terms and replacing $(n)$ by $(n+2)$ in the first sum, we assume the form

$$
\begin{gathered}
\sum_{n=0}^{\infty} a^{n+2} \frac{x^{n \alpha}}{\Gamma(n \alpha+1)}-\sum_{n=0}^{\infty} a^{n} \frac{x^{n \alpha}}{\Gamma(n \alpha+1)}=0, \\
\sum_{n=0}^{\infty}\left(a^{n+2}-a^{n}\right) \frac{x^{n \alpha}}{\Gamma(n \alpha+1)}=0 .
\end{gathered}
$$

With the Coefficient of $x^{n \alpha}$ equal to zero and identifying the coefficients, we obtain recursive

$$
a^{n+2}=a^{n}
$$

Substituting into (3.2), we find that:

$$
y(x)=1+a \frac{x^{\alpha}}{\Gamma(\alpha+1)}+\frac{x^{2 \alpha}}{\Gamma(2 \alpha+1)}+a^{2} \frac{x^{3 \alpha}}{\Gamma(3 \alpha+1)}+\cdots
$$

If $a=1$, we can write the general solution in the Mittag-Leffler function form as

$$
y(x)=\sum_{n=0}^{\infty} \frac{x^{\alpha}}{\Gamma(n \alpha+1)}=E_{\alpha}\left(x^{\alpha}\right)
$$

which is the exact solution of the linear fractional differential equation (4.9). 
Example 4.3. Consider the fractional differential equation [43]

$$
\frac{d^{2 \alpha} y}{d x^{2 \alpha}}+\frac{d^{\alpha} y}{d x^{\alpha}}-2 y=0
$$

By using (3.2) and (3.4) into (4.15) we find

$$
\sum_{n=2}^{\infty} a^{n} \frac{x^{(n-2) \alpha}}{\Gamma((n-2) \alpha+1)}+\sum_{n=1}^{\infty} a^{n} \frac{x^{(n-1) \alpha}}{\Gamma((n-1) \alpha+1)}-2 \sum_{n=0}^{\infty} a^{n} \frac{x^{n \alpha}}{\Gamma(n \alpha+1)}=0
$$

Combining the alike terms and replacing $(n)$ by $(n+2)$ in the first sum, we assume the form

$$
\begin{gathered}
\sum_{n=0}^{\infty} a^{n+2} \frac{x^{n \alpha}}{\Gamma(n \alpha+1)}+\sum_{n=0}^{\infty} a^{n+1} \frac{x^{n \alpha}}{\Gamma(n \alpha+1)}-2 \sum_{n=0}^{\infty} a^{n} \frac{x^{n \alpha}}{\Gamma(n \alpha+1)}=0 \\
\sum_{n=0}^{\infty}\left(a^{n+2}+a^{n+1}-2 a^{n}\right) \frac{x^{n \alpha}}{\Gamma(n \alpha+1)}=0
\end{gathered}
$$

With the coefficient of $x^{n \alpha}$ equal to zero and identifying the coefficients, we obtain recursive

$$
a^{n+2}=2 a^{n}-a^{n+1}
$$

Substituting into (3.2), we find that:

$$
y(x)=1+a \frac{x^{\alpha}}{\Gamma(\alpha+1)}+(2-a) \frac{x^{2 \alpha}}{\Gamma(2 \alpha+1)}+(a-2) \frac{x^{3 \alpha}}{\Gamma(3 \alpha+1)}+\cdots
$$

If $a=1$, we can write the general solution in the Mittag-Leffler function form as

$$
y(x)=\sum_{n=0}^{\infty} \frac{x^{\alpha}}{\Gamma(n \alpha+1)}=E_{\alpha}\left(x^{\alpha}\right)
$$

which is the solution of the linear fractional differential equation (4.15).

\section{Conclusions}

A new generalization of the Mittag-Leffler function method has been developed for linear differential equations with fractional derivatives. The new generalization is based on the Caputo fractional derivative. It may be concluded that this technique is very powerful and efficient in finding the analytical solutions for a large class of linear differential equations of fractional order. 
International Journal of Differential Equations

\section{References}

[1] Y. I. Babenko, Heat and Mass Transfer, Chemia, Leningrad, Germany, 1986.

[2] M. Caputo and F. Mainardi, "Linear models of dissipation in anelastic solids," La Rivista del Nuovo Cimento, vol. 1, no. 2, pp. 161-198, 1971.

[3] R. Gorenflo and F. Mainardi, "Fractional calculus: integral and differential equations of fractional order," in Fractals Fractional Calculus in Continuum Mechanics, A. Carpinteri and F. Mainar, Eds., pp. 223-276, Springer, New York, NY, USA, 1997.

[4] R. Gorenflo and R. Rutman, "On ultraslow and intermediate processes," in Transform Methods and Special Functions, P. Rusev, I. Dimovski, and V. Kiryakova, Eds., pp. 61-81, Science Culture Technology Publishing, Singapore, 1995.

[5] F. Mainardi, "Fractional relaxation and fractional diffusion equations, mathematical aspects," in Proceedings of the 12th IMACS World Congress, W. F. Ames, Ed., vol. 1, pp. 329-332, Georgia Tech Atlanta, 1994.

[6] F. Mainardi, "Fractional calculus: some basic problems in continuum and statistical mechanics," in Fractals and Fractional Calculus in Continuum Mechanics, A. Carpinteri and F. Mainardi, Eds., pp. 291348, Springer, New York, NY, USA, 1997.

[7] S. G. Samko, A. A. Kilbas, and O. I. Marichev, Fractional Integrals and Derivatives: Theory and Applications, Gordon and Breach Science Publishers, New York, NY, USA, 1993.

[8] H. Beyer and S. Kempfle, "Definition of physically consistent damping laws with fractional derivatives," Journal of Applied Mathematics and Mechanics, vol. 75, pp. 623-635, 1995.

[9] S. Kempfle and H. Beyer, "Global and causal solutions of fractional differential equations," in Proceedings of the 2nd International Workshop on Transform Methods and Special Functions, pp. 210-216, Science Culture Technology Publishing, Varna, Bulgaria, 1996.

[10] R. L. Bagley, "On the fractional order initial value problem and its engineering applications," in Fractional Calculus and Its Applications, K. Nishimoto, Ed., pp. 12-20, College of Engineering, Nihon University, Tokyo, Japan, 1990.

[11] A. A. Kilbas and M. Saigo, "On mittag-leffler type function, fractional calculus operators and solutions of integral equations," Integral Transforms and Special Functions, vol. 4, no. 4, pp. 355-370, 1996.

[12] Y. F. Luchko and H. M. Srivastava, "The exact solution of certain differential equations of fractional order by using operational calculus," Computers and Mathematics with Applications, vol. 29, no. 8, pp. 73-85, 1995.

[13] M. W. Michalski, “On a certain differential equation of non-integer order," Zeitschrift fur Analysis und ihre Anwendungen, vol. 10, pp. 205-210, 1991.

[14] M. W. Michalski, Derivatives of Non-integer Order and Their Applications, vol. 328 of Dissertationes Mathematicae, Polska Akademia Nauk, Institut Matematyczny, Warszawa, Poland, 1993.

[15] K. S. Miller and B. Ross, An Introduction to the Fractional Calculus and Fractional Differential Equations, John Wiley \& Sons, New York, NY, USA, 1993.

[16] I. Podlubny, "Solution of linear fractional differential equations with constant coefficients," in Transform Methods and Special Functions, P. Rusev, I. Dimovski, and V. Kiryakova, Eds., pp. 227-237, Science Culture Technology Publishing, Singapore, 1995.

[17] S. B. Hadid and Y. F. Luchko, "An operational method for solving fractional differential equations of an arbitrary real order," Pan-American Mathematical Journal, vol. 6, pp. 57-73, 1996.

[18] J. Padovan, "Computational algorithms for FE formulations involving fractional operators," Computational Mechanics, vol. 2, no. 4, pp. 271-287, 1987.

[19] S. Momani, "Non-perturbative analytical solutions of the space- and time-fractional Burgers equations," Chaos, Solitons and Fractals, vol. 28, no. 4, pp. 930-937, 2006.

[20] S. Momani and Z. Odibat, "Analytical solution of a time-fractional Navier-Stokes equation by Adomian decomposition method," Applied Mathematics and Computation, vol. 177, no. 2, pp. 488-494, 2006.

[21] Z. M. Odibat and S. Momani, “Approximate solutions for boundary value problems of time-fractional wave equation," Applied Mathematics and Computation, vol. 181, no. 1, pp. 767-774, 2006.

[22] S. Momani, "An explicit and numerical solutions of the fractional KdV equation," Mathematics and Computers in Simulation, vol. 70, no. 2, pp. 110-118, 2005. 
[23] S. Momani and Z. Odibat, "Analytical approach to linear fractional partial differential equations arising in fluid mechanics," Physics Letters, Section A, vol. 355, no. 4-5, pp. 271-279, 2006.

[24] S. Momani and Z. Odibat, "Numerical comparison of methods for solving linear differential equations of fractional order," Chaos, Solitons and Fractals, vol. 31, no. 5, pp. 1248-1255, 2007.

[25] J. H. He, "Approximate analytical solution for seepage flow with fractional derivatives in porous media," Computer Methods in Applied Mechanics and Engineering, vol. 167, no. 1-2, pp. 57-68, 1998.

[26] Z. M. Odibat and S. Momani, "Application of variational iteration method to nonlinear differential equations of fractional order," International Journal of Nonlinear Sciences and Numerical Simulation, vol. 7, no. 1, pp. 15-27, 2006.

[27] Z. Odibat and S. Momani, "Modified homotopy perturbation method: application to quadratic Riccati differential equation of fractional order," Chaos, Solitons and Fractals, vol. 36, no. 1, pp. 167-174, 2008.

[28] S. Momani and Z. Odibat, "Comparison between the homotopy perturbation method and the variational iteration method for linear fractional partial differential equations," Computers and Mathematics with Applications, vol. 54, no. 7-8, pp. 910-919, 2007.

[29] J. D. Munkhammar, "Fractional calculus and the Taylor-Riemann series," Undergraduate Mathematics Journal, vol. 6, 2005.

[30] R. L. Magin, "Fractional calculus in bioengineering," Critical Reviews in Biomedical Engineering, vol. 32, no. 1, pp. 1-104, 2004.

[31] R. L. Magin, "Fractional calculus in bioengineering, part 2," Critical Reviews in Biomedical Engineering, vol. 32, no. 2, pp. 105-193, 2004.

[32] R. L. Magin, "Fractional calculus in bioengineering, part 3," Critical Reviews in Biomedical Engineering, vol. 32, no. 3-4, pp. 195-377, 2004.

[33] K. B. Oldham and J. Spanier, The Fractional Calculus, Academic Press, New York, NY, USA, 1974.

[34] S. G. Samko, A. A. Kilbas, and O. I. Marichev, Fractional Integrals and Derivatives-Theory and Applications, Gordon and Breach Science Publishers, Longhorne, Pa, USA, 1993.

[35] S. Z. Rida, A. M. A. El-Sayed, and A. A. M. Arafa, "Effect of bacterial memory dependent growth by using fractional derivatives reaction-diffusion chemotactic model," Journal of Statistical Physics, vol. 140, no. 4, pp. 797-811, 2010.

[36] A. M. A. El-Sayed, S. Z. Rida, and A. A. M. Arafa, "On the Solutions of the generalized reactiondiffusion model for bacteria growth," Acta Applicandae Mathematicae, vol. 110, pp. 1501-1511, 2010.

[37] S. Z. Rida, A. M. A. El-Sayed, and A. A. M. Arafa, "On the solutions of time-fractional reactiondiffusion equations," Communications in Nonlinear Science and Numerical Simulation, vol. 15, no. 12, pp. 3847-3854, 2010.

[38] S. Z. Rida, A. M. A. El-Sayed, and A. A. M. Arafa, "A Fractional Model for Bacterial Chemoattractant in a Liquid Medium," Nonlinear Science Letters A, vol. 1, no. 4, pp. 415-420, 2010.

[39] A. M. A. El-Sayed, S. Z. Rida, and A. A. M. Arafa, "Exact solutions of fractional-order biological population model," Communications in Theoretical Physics, vol. 52, no. 6, pp. 992-996, 2009.

[40] A. M. A. El-Sayed, S. Z. Rida, and A. A. M. Arafa, "On the solutions of time-fractional bacterial chemotaxis in a diffusion gradient chamber," International Journal of Nonlinear Sciences, vol. 7, no. 4, p. 485, 2009.

[41] S. Z. Rida, H. M. El-Sherbiny, and A. A. M. Arafa, "On the solution of the fractional nonlinear Schrödinger equation," Physics Letters A, vol. 372, no. 5, pp. 553-558, 2008.

[42] I. Podlubny, Fractional Differential Equations: An Introduction to Fractional Derivatives, Fractional Differential Equations, Some Methods of Their Solution and Some of Their Applications to Methods of Their Solution and Some of Their Applications, Academic Press, New York, NY, USA, 1999.

[43] Z. M. Odibat and N. T. Shawagfeh, "Generalized Taylor's formula," Applied Mathematics and Computation, vol. 186, no. 1, pp. 286-293, 2007.

[44] A. A. Kilbas, M. Rivero, L. Rodríguez-Germá, and J. J. Trujillo, “ $\alpha$-analytic solutions of some linear fractional differential equations with variable coefficients," Applied Mathematics and Computation, vol. 187, no. 1, pp. 239-249, 2007. 


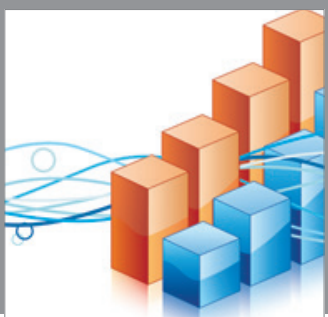

Advances in

Operations Research

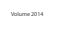

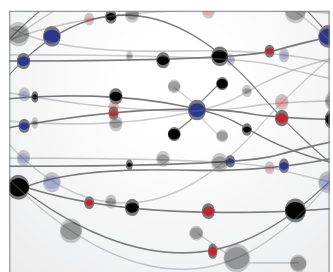

\section{The Scientific} World Journal
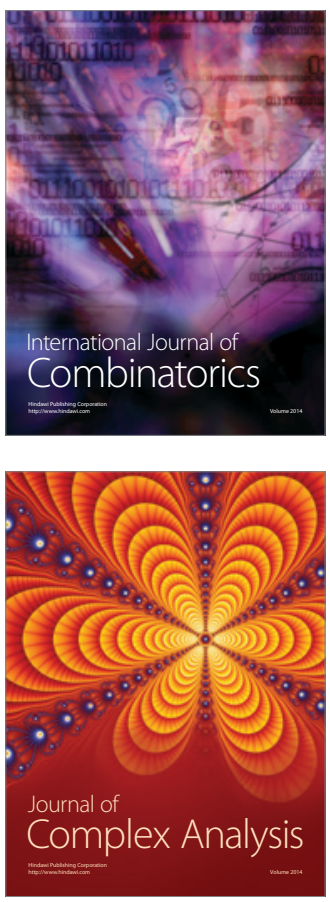

International Journal of

Mathematics and

Mathematical

Sciences
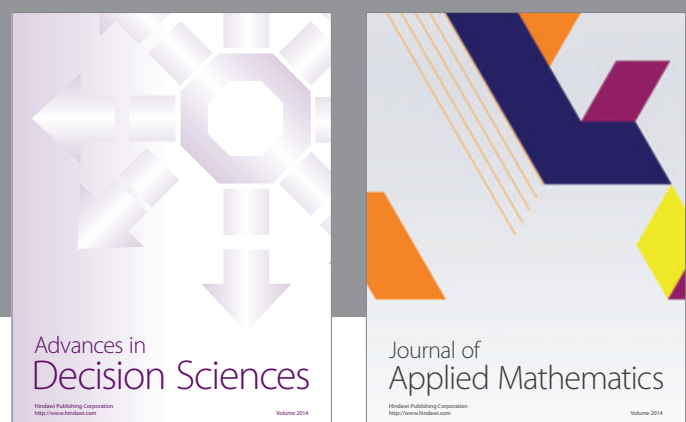

Journal of

Applied Mathematics
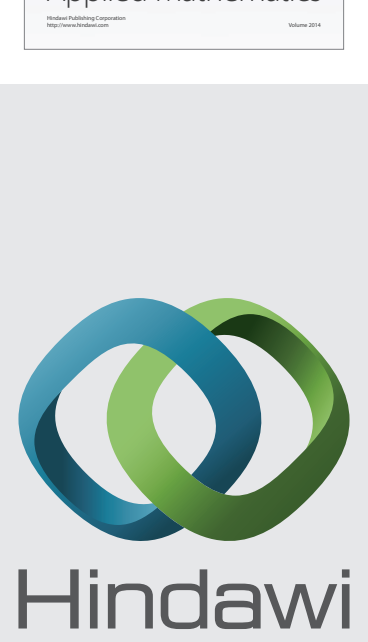

Submit your manuscripts at http://www.hindawi.com
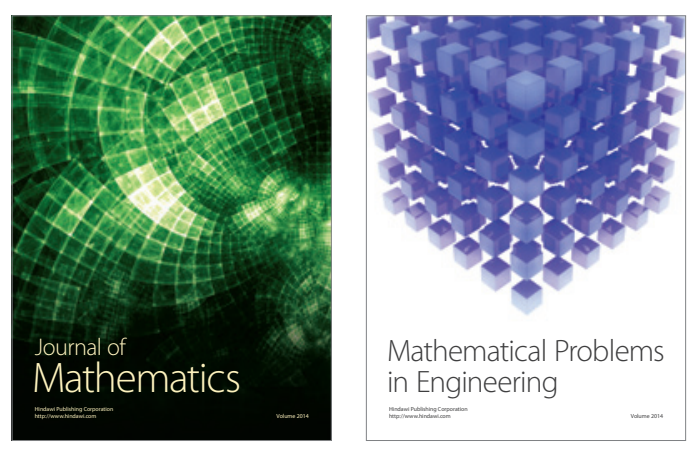

Mathematical Problems in Engineering
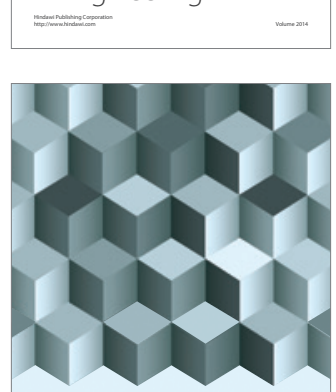

Journal of

Function Spaces
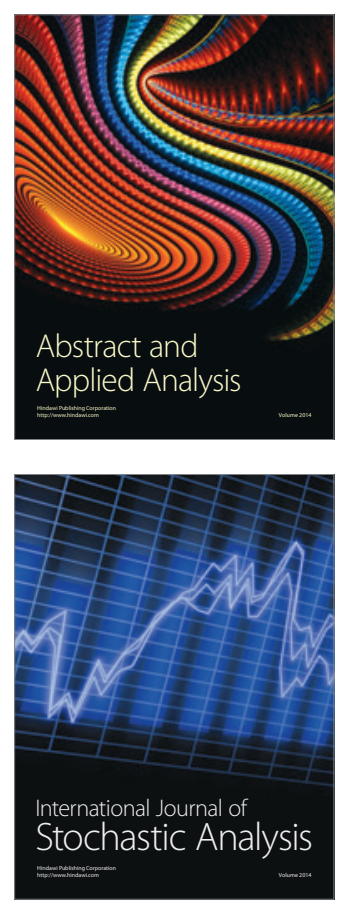

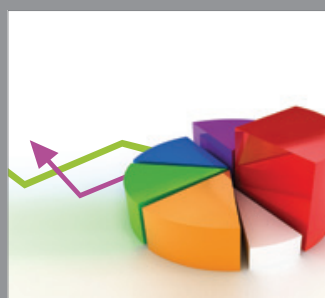

ournal of

Probability and Statistics

Promensencen
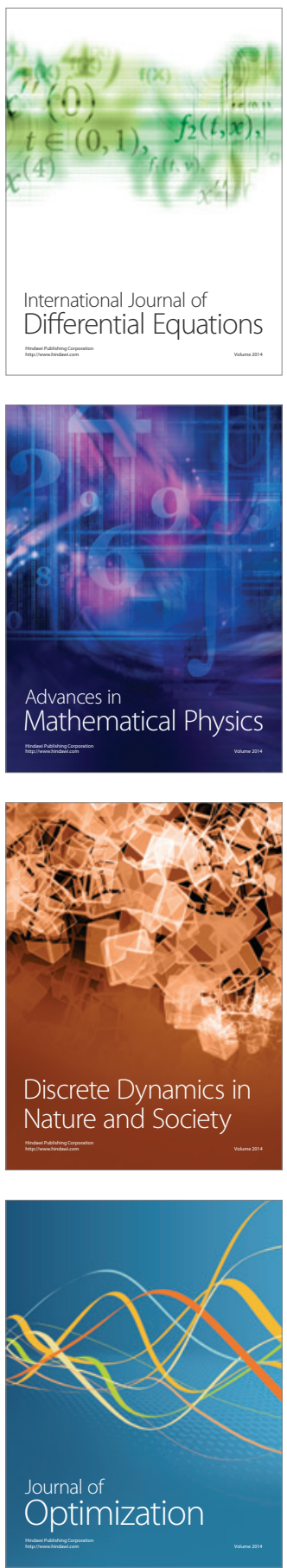